\title{
CHANGES IN CELLULAR AUTOPHAGIC CAPACITY DURING AZASERINE-INITIATED PANCREATIC CARCINOGENESIS*
}

\author{
S. Tóth, KrisZtina Nagy, Z. PÁlfia and G. RÉZ** \\ Department of General Zoology, Eötvös Loránd University, \\ P.O. Box 330, H-1445 Budapest, Hungary
}

(Received: January 10, 2001; accepted: March 2, 2001)

\begin{abstract}
Growth regulation is a crucial event in tumour progression. Surprisingly, relatively few papers have dealt with the catabolic side of regulation, and there are practically no data regarding the autophagic process during tumour development. We approach this problem by morphometrical investigation into the possible changes of autophagic activity during the progression of rat pancreatic adenocarcinoma induced by azaserine. In the present study, autophagic capacity of the azaserine-induced premalignant and malignant cells were characterised and compared to the respective host tissue cells of the rat pancreas and to the acinar cells in other stages of tumour development. Using vinblastine (VBL) as an enhancer, and cycloheximide (CHI) as an inhibitor of autophagic segregation we observed that autophagic capacity of premalignant cells (month 6 and 10 after initiation) is much higher than in the host tissue cells. We found a sharp decrease in self-digesting capacity in adenocarcinoma cells (month 20) where VBL induced a minimal accumulation of autophagic vacuoles which was, surprisingly, not inhibited by CHI, i.e. the CHIsensitive regulatory step was lost. The changes in autophagic capacity are probably associated to specific steps of tumour progression in our system.
\end{abstract}

Keywords: Autophagy - pancreas - carcinogenesis - tumour progression - morphometry

\section{INTRODUCTION}

Changes in growth regulation is a crucial event in cancer development. In this respect, autophagic function of the tumour cells is of interest because changes in cellular self-digesting capacity during progression may contribute to tumour development or regression. Autophagy may have a significant role also in tumour cell death $[4,19]$.

Most studies showed that degradation of endogenous proteins in tumour cells is downregulated compared to normal cells $[1-3,6,8,15,20-22]$, although increased activity was also measured under some growth circumstances [23]. Cellular autophagy in premalignant and malignant cells in vitro was shown to be supressed $[8,21]$. However, data for autophagic activity or capacity in premalignant and malignant cells throughout tumour progression in vivo are almost completely lacking.

\footnotetext{
*Dedicated to Professor János Kovács on the occasion of his 70th birthday.

**Corresponding author; e-mail: grez@cerberus.elte.hu
} 
It is possible that autophagy takes an important part in development of tumour and autophagy has an essential role in regression and progression of malignancy, and nothing is known about the timing of changes in regulation of autophagy during tumour progression. An extension of research efforts to this field seems desirable from both cells biological and pathological point of view.

To approach this problem, we applied a pancreatic carcinogenesis model $[9,10$, 25], in which the target cell is the exocrine pancreatic acinar cell of the rat. Using azaserine as initiator and a pancreatotrophic diet as promoter it is possible to produce several tumours in the same pancreas. We detected the first premalignant atypical acinar cell nodules (AACN) 5 months after initiation. Later, at months $10-15$, benign pancreatic adenomas, and from months 18 well-differentiated adenocarcinomas in situ (CIS) are present in the same animal $[9,10]$.

To investigate the autophagic capacity of the premalignant and malignant cells, we used electron microscopic morphometrical measurement of volume changes of the autophagic compartment $[17,18]$, i.e. its expansion upon stimulation and regression upon inhibition of autophagic vacuole (AV) formation. Using vinblastine (VBL) as an enhancer [12,18] and cycloheximide (CHI) as an inhibitor [11-13] of autophagic segregation, we estimated the rate of segregation and digestive capacity of tumour cells in different stages of progression.

In our previous study [17] we reported that the autophagic capacity highly increased in premalignant AACN cells. In the present paper we show preliminary results of our study in which changes of autophagic capacity were followed throughout the whole tumour progression. Our results show that the elevated autophagic capacity of premalignant cells sharply decrease in the tumour cells of the malignant stage concomitantly with the dissapearance of the CHI-sensitive point of autophagic regulation.

\section{MATERIALS AND METHODS}

\section{Animals and treatments}

Five-week-old male Wistar rats (purchased from Humán Co., Gödöllő, Hungary) were fed ad libitum with a standard commercial laboratory diet (Charles River Hungary Ltd., Budapest, Hungary) for 2 weeks when their body weight reached $200-250 \mathrm{~g}$. Then the animals received a single carcinogen injection (i.p., $30 \mathrm{mg} / \mathrm{kg}$ b.w.) of aqueous azaserine (Sigma-Aldrich Ltd., Budapest, Hungary) solution (30 $\mathrm{mg} / \mathrm{ml}$ ) [25] and their food was changed to the semisynthetic pancreatotrophic raw soybean flour-based diet $[17,25]$. The rats had free access to this diet and water throughout the experimental period.

Groups of 12 animals were used 5, 6, 8, 10, 15 and 20 month after the azaserine injection. Each group was subdivided into three subgroups, i.e. untreated, VBL-treated and VBL + CHI-treated animals. The latter two subgroups received a single intraperitoneal injection of $5 \mathrm{mg} / \mathrm{kg}$ b.w. aqueous $(5 \mathrm{mg} / \mathrm{ml})$ vinblastine sulphate 
(Gedeon Richter Pharmaceutical Factory Co., Budapest, Hungary). The VBL-treated subgroup was sacrificed 90 minutes after the injection, when the VBL + CHI-treated animals received aqueous CHI (Sigma-Aldrich Ltd., Budapest, Hungary) injection $(0.5 \mathrm{mg} / \mathrm{kg}$ b.w. in $0.5 \mathrm{mg} / \mathrm{ml}$ solution) for an additional 20 minutes. The effect of the drugs on the tumour cells was compared with the reaction of tumour bearing host tissue. We have shown recently [17] that azaserine-treated host pancreatic cells react to VBL and CHI much the same way as controls without azaserine treatment do. The rats were sacrificed by decapitation under ether anesthesia and their pancreata were processed for light and electron microscopy.

\section{Morphological and morphometrical methods}

Tissue pieces were fixed for $2 \mathrm{~h}$ in $0.1 \mathrm{M}$ sodium cacodylate-HCl-buffered (pH 7.2) $1 \%$ glutaraldehyde and post-fixed in cacodylate-buffered $1 \%$ osmium tetrahydroxide acid solution for $1 \mathrm{~h}$. This was followed by block contrasting in $2 \%$ aquaeous uranyle acetate and embedding in Durcupan (Fluka Chemie AG, Buchs, Switzerland).

Morphometrical measurements were carried out by the point counting method as described by Weibel [24]. Semithin sections of the tissue samples were made and stained either with toluidine blue-Azure II, or methylene blue-basic fuchsin mixture. AACNs and the surrounding host tissue cells were distinguished in the light microscope by their differential morphology and basophilic staining. Adenomas of macroscopic size were isolated from the fresh tissue and processed individually for electron microscopy. Four rats were used in each experimental group. Four host tissue, and at least four tumour samples were taken for ultrathin sectioning from each animal. Five electron micrographs were taken per sample at a primary magnification of 4000 in a Jeol JEM-100 CX II electron microscope operated at $60 \mathrm{kV}$. The cytoplasmic volume fractions (CVF) of the compartments and subcompartments of interest were expressed as percentage of the cytoplasmic volume. Regarding the uneven distribution of the primary point counts, the Mann-Whitney $u$-test, rather than Student's $t$-test was used for statistical evaluation of the data.

The autophagic compartment (total autophagic vacuoles, $\mathrm{AV}_{\mathrm{t}}$ ) was divided into three subcompartments according to functional morphological criteria. Early autophagic vacuoles $\left(\mathrm{AV}_{1}\right)$ contain seemingly undegraded material, whereas advanced $\mathrm{AVs}\left(\mathrm{AV}_{2}\right)$ exhibit degrading substances of recognizable origin. Late AVs $\left(\mathrm{AV}_{3}\right)$ are usually large-sized secondary lysosomes enclosing highly degraded substances whose origin can no longer be determined. In the present study cytoplasmic volume fraction (CVF) of AVs was calculated by summation of CVF of all subcompartments, thus $\mathrm{AV}_{\mathrm{t}}=\mathrm{AV}_{1}+\mathrm{AV}_{2}+\mathrm{AV}_{3}$. The rate of expansion $\left(\mathrm{V}_{\text {exp }}\right.$ in CVF \% min $\left.{ }^{-1}\right)$ is the AV cytoplasmic volume fraction expanded in the 90 min after VBL treatment, the rate of regression $\left(\mathrm{V}_{\mathrm{reg}}\right.$ in $\left.\mathrm{CVF} \% \mathrm{~min}^{-1}\right)$ is the $\mathrm{AV}$ cytoplasmic volume fraction regressed in the 20 min after $\mathrm{CHI}$ treatment. The rate of segregation $\left(\mathrm{V}_{\text {seg }}\right.$ in $\mathrm{CVF} \%$ $\min ^{-1}$ ) was calculated by summation of $V_{\text {exp }}$ and $V_{\text {reg }}$. 


\section{RESULTS}

We reported recently [17] that raw soya diet and azaserine treatment has no significant effect on autophagic function of pancreatic acinar cell, so in our investigation, tumour-bearing host tissue cell is a reliable control for the respective tumour cells. In untreated host control cells, level of cytoplasmic volume fraction of autophagic com-

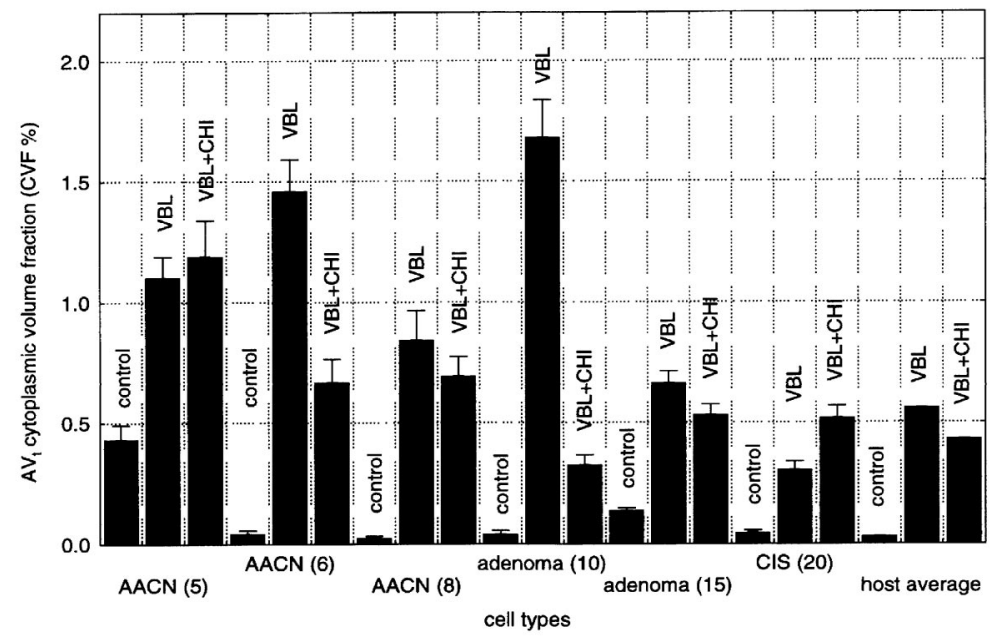

Fig. 1. Volume changes of total autophagic compartment $\left(\mathrm{AV}_{\mathrm{t}}\right)$ in premalignant and malignant cells after VBL and VBL + CHI treatment. Numbers in parentheses show the month after azaserine initiation. Since there is no significant difference in autophagic capacity between host tissue cells in different time points of tumor progression, host average were used to demonstrate the volume changes

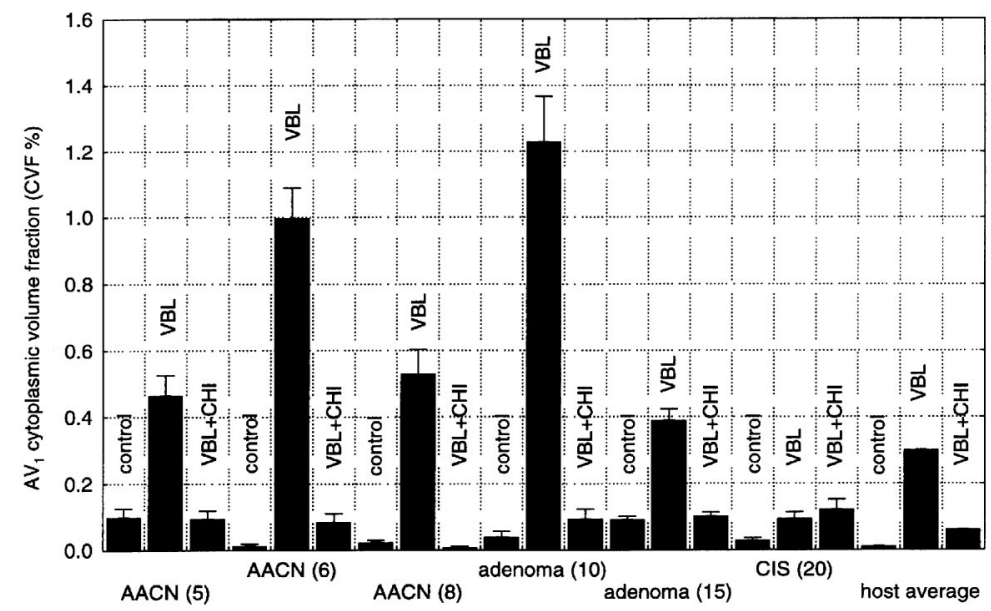

Fig. 2. Volume changes of early autophagic compartment $\left(\mathrm{AV}_{1}\right)$ in premalignant and malignant cells after VBL and VBL+CHI treatment. Numbers in parentheses show the month after azaserine initiation 
partment is very low, about $0.03 \%$. As it was expected, VBL treatment caused a significant enlargement of $\mathrm{AV}_{\mathrm{t}}$ after $90 \mathrm{~min}(0.55 \%$, Fig. 1). Upon CHI post-treatment, well-recognisable regression of $\mathrm{AV}$ compartment was shown only in $\mathrm{AV}_{1}$ subcompartment, in which the volume of this subtype decreased to that of the untreated control (from $0.30 \%$ to $0.03 \%$, Fig. 2). Volume of $\mathrm{AV}_{\mathrm{t}}$ decreased only slightly or not at all, the reason of which is the retardation caused by VBL in the regression of the $\mathrm{AV}_{2}$ subtype. Compared to untreated steady-state cells (where segregation and regression rates fall in the range $\left.0.003-0.008 \mathrm{CVF} \% \mathrm{~min}^{-1}\right)[7,13]$, an elevated segregational rate was found in VBL-treated host tissue cells $\left(0.015 \mathrm{CVF} \% \mathrm{~min}^{-1}\right.$ in case of $\left.\mathrm{AV}_{1}\right)$. Rate of regression of AV compartment is about $0.006 \mathrm{CVF} \% \mathrm{~min}^{-1}$ (Fig. 3). These finding strengthen our oppinion $[12,17,18]$ that VBL exerted a dual influence: stimulation of segregation and retardation of further AV processing.

We found a very small autophagic compartment in premalignant and malignant cells $\left(\mathrm{AV}_{\mathrm{t}}=\sim 0.04 \%\right)$ at all time points but in month $5 \mathrm{AACN}(0.43 \%)$ and month 15 adenoma $(0.14 \%)$ cells. In month 5 AACN cells, an increased regressional rate $\left(0.021 \mathrm{CVF} \% \mathrm{~min}^{-1}\right)$ was obtained after $20 \mathrm{~min}$ CHI treatment.

Premalignant and malignant cells at each time point of progression have reacted to VBL-treatment in a different way. The largest AV compartment was induced in the month 6 AACN (1.46\%) and month 10 adenoma (1.63\%) cells (Fig. 2). CIS cells in month 20 had a weak and slow reaction to VBL-treatment $\left(\mathrm{AV}_{\mathrm{t}}\right.$ is about $0.3 \%$, lower, than that measured in the host tissue cells). In all cell types, the largest subcompartment of $\mathrm{AV}_{\mathrm{t}}$ was $\mathrm{AV}_{1}$, but in month $6 \mathrm{AACN}$ and month 10 adenoma cells, volume of this subcompartment was extremely high $(1.0 \%$ and $1.23 \%$, respectively, Fig. 2$)$.

Upon CHI post-treatment, significant decrease of AV compartment was observed in the premalignant cells at each time point after initiation (Figs 1,2), except in the

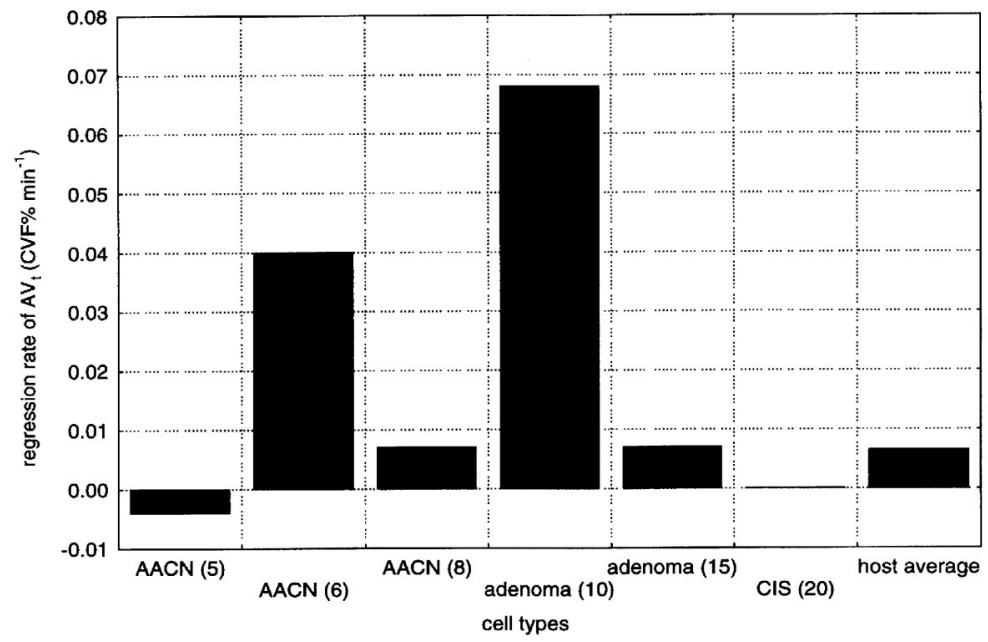

Fig. 3. The rate of $\mathrm{AV}_{\mathrm{t}}$ regression in pancreatic acinar cells of different stage of tumour progression. Numbers in parentheses show the month after azaserine initiation 


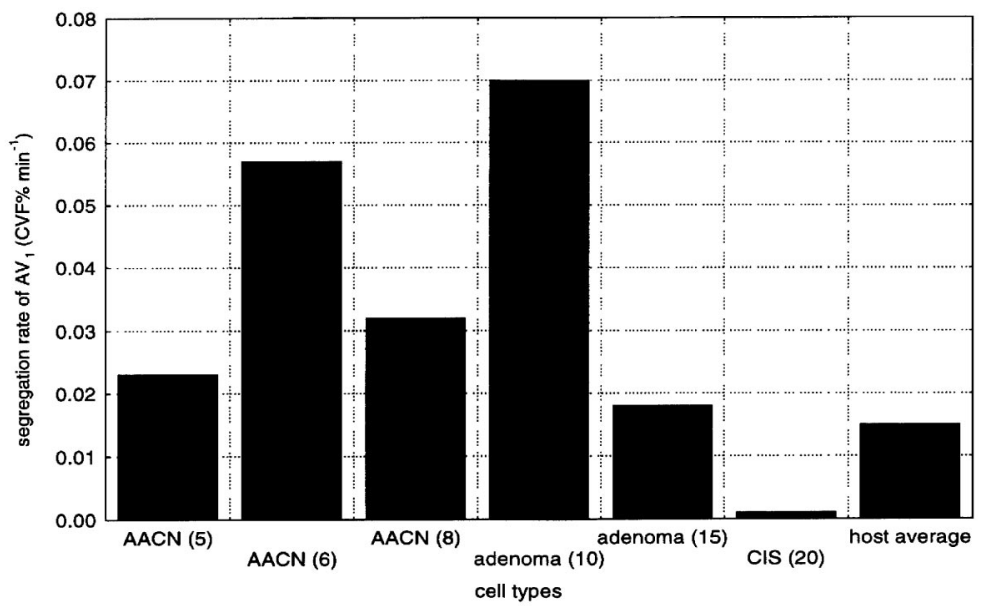

Fig. 4. The rate of autophagic segregation ( $\mathrm{AV}_{1}$ formation) in pancreatic acinar cells of different stage of tumour progression. Numbers in parentheses show the month after azaserine initiation

5-months AACN cells, where the volume of late $\mathrm{AVs}$ has remained high, so that the volume of $\mathrm{AV}_{\mathrm{t}}$ was almost unchanged (similar to that we observed in host tissue cells). Surprisingly, $\mathrm{CHI}$ did not cause either $\mathrm{AV}_{\mathrm{t}}$ or $\mathrm{AV}_{1}$ regression in CIS cells (Figs $1,2)$.

Calculated data for regression and segregation rate (Figs 3,4) show that in the premalignant stages (month 6 and 10) autophagic capacity increased 5-10 and 10-20 fold when compared to other cell types in our system [17] or to steady-state cells [7, 13], respectively. However, month 20 CIS malignant cells showed a very low autophagic capacity (only about half of that measured in steady-state cells).

\section{DISCUSSION}

On the basis of data reported here we conclude that autophagic capacity in premalignant and malignant acinar cells shows characteristical changes in the course of progression of pancreatic acinar cell adenocarcinoma. In the same time there is no significant change in autophagic activity/capacity of host cells during tumor development.

Pancreatic acinar cell adenocarcinoma develops through specific stages $[9,10]$. Numerous atypical acinar cell nodules arise from normal acini after induction, but only a few of them develop to adenomas. There may be a number of effects to influence this premalignant progression. Our data clearly indicate the existence of significant differences in autophagic activity/capacity between the 5-, 6- and 8-month-old AACN cells. An increased autophagic activity with a moderate further autophagic capacity was measured in the month $5 \mathrm{AACN}$, whereas low autophagic activity with highly increased autophagic capacity belongs to the 6 month AACN stage. There are 
at least three possible explanations for this oscillation of autophagic activity/capacity with progression time.

Firstly, it is possible that an increased autophagy is necessary to eliminate a part of AACNs. In some cases tumour cell death starts with autophagy $[4,19]$. Secondly, the step from a previous stage of progression into the next one may require a transiently increased autophagic degradation, analogously to remodelling (phenotype change) of cells of cycling function [5] or changing workload [14]. Thirdly, cellular autophagy may be associated to growth cessation [14] in some tumours in stationary stage. It is noteworthy that there exist a coincidence in time of autophagic capacity increase and appearance $[9,10]$ of adenomas. Both events occur around the 5th-6th month of progression, which may provide a possible explanation for the increased autophagic activity of month $5 \mathrm{AACN}$ cells, and the highly increased segregation and degradation capacity of month $6 \mathrm{AACN}$ cells. Increased autophagic activity at month 5 may reflect an ungoing remodelling or a stationary phase (for apoptotic cells were not found at this stage). Increased autophagic capacity with latent activity may render tumour cells highly sensitive to either internal or external stimulants of lysosomal self-digestion, which may be a prerequisite for the switch-on of either remodelling or autophagic cell death.

In contrast with earlier stages, month 8 AACNs exhibit only a moderate degradation capacity, which may be attributed to a growth phase as growing tumour cells were characterized by a low protein turnover and autophagy [15, 23]. There is another coincidence of a tumour progression step and high autophagic capacity in the adenomas in month 10, when the first in situ adenocarcinomas $[9,10]$ appear. According to the aforelaunched speculation, month 10 adenomas may be ready for the next remodellings: either for the transformation to CIS or to become "established adenomas" $[9,10]$ that are supposed to be non- or slowly-growing benign tumours. And indeed, the next month 15 adenomas exhibited a moderately increased autophagic activity and a slight further autophagic capacity.

The most interesting changes were observed in the 20-month time point of progression. The results are unusual as compared to data measured and published before. Vinblastine had only a slight but persistent influence on well-differentiated adenocarcinoma cells as cycloheximide had no effect on segregation. The fact that protein synthesis inhibitors emetine, puromycin [11] and CHI [11-13] are in vivo segregation inhibitors raises the possibility that de novo protein synthesis is needed for segregation. Anyway, the loss of CHI-sensitive point in regulation of autophagy seems to be lost in azaserine-induced pancreatic adenocarcinoma. The residual VBL-inducible segregation may be caused by a CHI-independent alternative pathway.

Summarising our results, we found a striking increase of autophagic capacity in premalignant cells. This observation is contradictory to that published by others [21, 22]. The difference might be caused by the different experimental conditions and/or cell type used in different studies. In malignant cells, however, we observed a very low autophagic capacity. This finding is in line with the reported low autophagic activity $[8,21]$ in other malignant tumor cell types. Our results show that probably a first specific step of progression exists in the stage of AACNs when highly elevated 
autophagic capacity may be required for tumor development, and a second one at the transition from the premalignant (adenoma) to the malignant (CIS) stage, when decrease of autophagic capacity and loss of $\mathrm{CHI}$-sensitive regulation is to occur.

\section{ACKNOWLEDGEMENTS}

We would like to thank Sarolta Pálfia, Ágnes Keserü and Mariann Saródy for their excellent technical assistance. This study was partly supported by a Copernicus Contract (CIP-ACT 930210) of the European Union.

\section{REFERENCES}

1. Ahlberg, J., Yucel, T., Eriksson, L., Glaumann, H. (1987) Characterization of the proteolytic compartment in rat hepatocyte nodules. Virchows Arch. B. Cell Path. 53, 79-88.

2. Baccino, F. M., Tessitore, L., Bonelli, G. (1984) Control of protein degradation and growth phase in normal and neoplastic cells. Toxicol. Pathol. 12, 281-287.

3. Boyer, M. J., Tannock, I. F. (1993) Lysosomes, lysosomal enzymes and cancer. Adv. Canc. Res. 60 , 269-291.

4. Bursch, W., Ellinger, A., Kienzl, H., Török, L., Pandey, S., Sikorska, M. Walker, R., Hermann, R. S. (1996) Active cell death induced by the anti-estrogens tamoxifen and ICI 164384 in human mammary carcinoma cells (MCF-7) in culture: the role of autophagy. Carcinogenesis 17, 1595-1607.

5. Farguhar, M. G. (1969) Lysosome function in regulating secretion: disposal of secretory granules in cells of the anterior pituitary gland. In: Dingle, J. T., Fell, H. B. (eds) Lysosomes in biology and pathology. North-Holland Publ. Co., Amsterdam-London, Vol. 2, pp. 462-482.

6. Gunn, J. M., Clank, M. G., Knowles, S. E., Hopgood, M. F., Ballard, F. J. (1977) Reduced rates of proteolysis in transformed cells. Nature 266, 58-60.

7. Kovács, J. (1983) Regression of autophagic vacuoles in seminal vesicle cells following cycloheximide treatment. Exp. Cell Res. 144, 231-234.

8. Kisen, G. X., Tessitore, L., Costelli, P., Gordon, P. B., Schwarze, P. E., Baccino, F. M., Seglen, P. O. (1993) Reduced autophagic activity in primary rat hepatocellular carcinoma and ascites hepatoma cells. Carcinogenesis 14(2), 2501-2505.

9. Longnecker, D. S., Roebuck, B. D., Yager, J. D., Lilja, H. S., Sigmund, B. (1981) Pancreatic carcinoma in azaserine-treated rats (induction, classification and dietary modulation of incidence). Cancer $47,1562-1572$.

10. Longnecker, D. S. (1986) Experimental models of exocrine pancreatic tumors. In: Go, V. L W. Gardner, J. D., Brooks, F. P., Lebenthal, E., Di Magno, E. P., Scheele, G. A. (eds) The exocrine pancreas biology, pathobiology and diseases. Raven Press, New York. pp. 443-458.

11. Oliva, O., László, L., Pálfia, Z., Réz, G. (1991) Translational inhibitors cycloheximide, emetin and puromycin inhibit cellular autophagy in mouse liver parenchymal and pancreatic acinar cells in vivo. Acta Morphologica Hung. 39(2), 79-85.

12. Oliva, O., Réz, G., Pálfia, Z., Fellinger, E. (1992) Dynamics of vinblastine-induced autophagocytosis in murine pancreatic acinar cells: influence of cycloheximide post-treatments. Exp. Molec. Pathol. 56, 76-86.

13. Papadopoulos, T., Pfeifer, U. (1986) Regression of rat liver autophagic vacuoles by locally applied cycloheximide. Lab. Invest. 54, 100-107.

14. Pfeifer, U. (1987) Functional morphology of the lysosomal apparatus. In: Glaumann, H., Ballard, F. J. (eds) Lysosomes, their role in protein breakdown. Academic Press, London, UK, pp. 3-59. 
15. Pfeifer, U., Tessitore, L., Bonelli, G., Baccino, F. M. (1988) Regulation of protein turnover versus growth state III. Growth cessation is associated with activation of autophagy in Yoshida ascites hepatoma AH-130. Virchows Arch. B Cell Pathol. 55, 3763-3769.

16. Punnonen, E. L., Reunanen, H. (1990) Effects of vinblastine, leucine, and histidine, and 3-methyladenine on autophagy in Ehrlich ascites cells. Exp. Mol. Pathol. 52, 87-97.

17. Réz, G., Tóth, S., Pálfia, Z. (1999) Cellular autophagic capacity is highly increased in azaserineinduced premalignant atypical acinar nodule cells. Carcinogenesis 20, 1893-1898.

18. Réz, G., Csák, J., Fellinger, E., László, L., Kovács, A. L., Oliva, O., Kovács, J. (1996) Time course of vinblastine-induced autophagocytosis and changes in the endoplasmic reticulum in murine pancreatic acinar cells: a morphometrical and biochemical study. Eur. J. Cell Biol. 71, 341-350.

19. Schulte-Hermann, R., Bursch, W., Grasl-Kraupp, B., Torok, L., Ellinger, A., Mullauer, L. (1995) Role of active cell death (apoptosis) in multi-stage carcinogenesis. Toxicol. Lett. 82-83, 143-148.

20. Schwarze, P. E., Seglen, P. O. (1985) Reduced autophagic activity, improved protein balance and enhanced in vitro survival of hepatocytes isolated from carcinogen-treated rats. Exp. Cell Res. 157, 15-28.

21. Seglen, P. O., Schwarze, P. E., Saeter, G. (1986) Changes in cellular ploidy and autophagic responsiveness during rat liver carcinogenesis. Toxicol. Pathol., 14, 342-348.

22. Seglen, P. O. (1997) DNA ploidy and autophagic protein degradation as determinants of hepatocellular growth and survival. Cell Biol. Toxicol. 13, 301-315.

23. Tessitore, L., Bonelli, G., Cecchini, G., Amenta, J. S., Baccino, F. M. (1987) Regulation of protein turnover versus growth state: ascites hepatoma as a model for studies both in the animal and in vitro. Arch. Biochem. Biophys. 255, 372-384.

24. Weibel, E. R. (1969) Stereological principles for morphometry in electron microscopic cytology. Int. Rev. Cytol., 26, 235-302.

25. Yager, J. D., Roebuck, B. D., Zurlo, J., Longnecker, D. S., Weselcouch, E. O., Wilpone, S. A. (1981) A single-dose protocol for azaserine initiation of pancreatic carcinogenesis in the rat. Int. J. Cancer 28, 601-606. 